\title{
Fixed Point Iteration-based Adaptive Control for a Delayed Differential Equation Model of Diabetes Mellitus*
}

\author{
Árpad Varga ${ }^{1}$, Levente Kovács ${ }^{2}$, György Eigner ${ }^{2}$, Dusan Kocur ${ }^{3}$, József K. Tar ${ }^{4}$
}

\begin{abstract}
In natural sciences, especially in life sciences, controller designers frequently meet the problem that though the controlled system is modeled by a set of nonlinearly coupled Ordinary Differential Equations (ODE) containing various independent variables, only a single control input is available by the use of which the propagation of only one variable has to be controlled. Normally the controlled state variable can be observed by direct measurements, while no sensors are available for obtaining information on the propagation of the other ones. Though in the possession of a reliable system model one has good odds to develop state observers, in the practice just the reliable model used to be missing. While the traditional control design methodologies normally need some state estimation, the Fixed Point Iteration-based (FPI) Adaptive Controller was developed to evade this difficulty. In this design instead modeling the effects of the propagation of the various state variables, these effects are directly observed and compensated on this basis. This approach can be used without structural modification if certain effects appear through some time-delay (Delayed Differential Equations - DDE). In many cases simple and effective models can be developed that contain only pure delay effects. In this paper it is shown that a recent DDE model of Diabetes Mellitus can be used in the FPI-based adaptive blood glucose concentration level control even if the available model is very imprecise. This statement is substantiated by numerical simulations.
\end{abstract}

\section{INTRODUCTION}

In natural sciences, especially in life sciences, controller designers frequently meet the problem that though the controlled system is modeled by a set of nonlinearly coupled ODEs containing various independent variables, only a single control input is available by the use of which the propagation of only one variable has to be controlled. In Classical Mechanics these systems are referred to as "underactuated" ones. For instance in anaesthesia control the measurable quantities that convey information on the state of the patient can be either the "Bispectral Index" [1], or a signal obtained by wavelet analysis [2] that can quantify the cortical activity

This project has received funding from the European Research Council (ERC) under the European Union's Horizon 2020 research and innovation programme (grant agreement No 679681).

1 The Authors is with the Applied Informatics and Applied Mathematics Doctoral School of Óbuda University, Budapest, Hungary varga.arpadekvk.uni-obuda.hu

2 The Authors are with the Physiological Controls Research Center within the Research, Innovation and Service Center of Óbuda University, Budapest, Hungary kovacseuni-obuda.hu, eigner.gyorgyenik.uni-obuda.hu

3 The Authors is with the Technical University of Kosice, Department of Electronics and Multimedia Communications, Kosice, Slovakia dusan.kocur@tuke.sk

${ }^{4}$ The Authors is with the Antal Bejczy Center for Intelligent Robotics within the Research, Innovation and Service Center of Óbuda University, Budapest, Hungary tar.jozsef@nik-obuda.hu of the patient. For the description of the same phenomenon different models of various complexity can be developed. For modeling the human glucose-insulin system the development simple "minimal model" by Bergman [3] was followed by more complex, multiple compartment models [4], [5] in which certain effects manifest themselves through the dynamic excitation of coupled subsystems, so some time is needed after the elapse of which these effects became "apparent".

A relatively simple modeling possibility is the replacement this complex mechanism by the introduction of pure timedelay in the model as in [6], [7], [21] in which a definite delay time is assumed as a constant parameter of the given model. However, in many cases the delay time cannot be constant. For instance, in object manipulation by teleoperation the various information packages travel along different routes on the communication media that may cause drastic, and stochastically time-varying effects [8]. Another typical example for short delays occurs whenever a mathematical algorithm formulated in continuous time is implemented as its discretized approximation by the use of digital controllers [9]-[11]. In this case the computational needed for the controller can cause some delay. The control of varying delay systems is an interesting problem. For switching systems [12]-[14] and neural network architectures [15] already significant results have been published.

The traditional controller design approaches normally need some "estimated state" of the controlled system. The traditional approaches as the Kalman filters [16] and the Luenberger observer [17] are designed for use in the case of Linear Time-invariant (LTI) systems. Considerable results were achieved in the field of the estimation of the state of nonlinear systems (e.g. [18]-[20]).

To evade the problem of state estimation in the case in which no reliable (formal) dynamic model is available, or in the case when the model is available but its application could conclude struggling with complicated formulae and computational burden adaptive approach was introduced [29], [30] in which the effects of the various terms in the model were not computed by the use of this model, its observed or estimated state variables and available approximate parameters. Instead of that it can be observed in the propagation of the directly observable and controlled variable, and after observation, it can be adaptively compensated. The great advantage of this approach is that no precise and well identified system model and parameters are needed for its use. This method can be used on the basis of a very primitive, approximate system model. In the same time, in general, it cannot be stated 
that such controller can be successful or precise enough. Its precision depends on the significance of the "omitted terms", and in the practice, simulation investigations have to done to answer this question. Its application is especially attractive in the case of a higher relative order control task, in which only a higher order time-derivative of the controlled quantity can be instantaneously set by the control signal through the effect chain of coupled dynamic variables.

The FPT based theorem has been successfully adapted to solve issues related to diabetes mellitus [34].

In the present paper the observer-based results published in [21] are considered with the aim of answering the question: is it possible to control the blood plasma glucose concentration in the case of the illness of Diabetes Mellitus by the simple FPI-based adaptive control? To this question the simulations provided positive and promising answer. In the sequel at first the DDE model of the phenomenon is analyzed. Following that the principles of the FPI-based adaptive control and the polynomial numerical differentiator applied in it is briefed. Finally simulation results are provided and the paper is closed by the conclusions.

\section{The DDE Model of Diabetes Mellitus}

The equations of motion of the model are given in (1), its variables and the parameters are expounded in Table I.

$$
\begin{aligned}
& \dot{G}(t)=-K_{x g i} G(t) I(t)+\frac{T_{g h}}{V_{G}}, \\
& \dot{I}(t)=-K_{x i} I(t)+\frac{T_{i G \max } f\left(G\left(t-\tau_{g}\right)\right)}{V_{I}}+ \\
& +\frac{S_{2}(t)}{V_{I} t_{\max , I}}, \\
& \dot{S}_{2}(t)=\frac{S_{1}(t)-S_{2}(t)}{t_{\max , I}}, \\
& \dot{S}_{1}(t)=-\frac{S_{1}(t)}{t_{\max , I}}+u(t) \\
& f(G) \stackrel{\text { def }}{=} \frac{\left(\frac{G}{G^{\star}}\right)}{1+\left(\frac{G}{G^{\star}}\right)} .
\end{aligned}
$$

It is evident that $u$ does not directly affect $\dot{G}$. To reveal the relative order of the possible control it can be noted that according to (1d) $\dot{S}_{1}(t)$ is directly influenced by $u$ as $\dot{S}_{1}(t)=u(t)+A d d_{1}$ in which $A d d_{1}$ denotes some "additional terms". The derivative of (1c) contains $\dot{S}_{1}(t)$, therefore $\ddot{S}_{2}(t)=u(t) / t_{\max , I}+A d d_{2}$. According to the derivative of (1b) $\dddot{I}$ contains $\ddot{S}_{2}(t)$, therefore $\dddot{I}(t)=$ $u(t) /\left(V_{I} t_{\max , I}^{2}\right)+A d d_{3}$. Finally, it can be observed that the third derivative of (1a) contains $\dddot{I}(t)$, therefore $\dddot{G}(t)=$ $-K_{x g i} G(t) u(t) /\left(V_{I} t_{\text {max }, I}^{2}\right)+A d d_{4}$. It is important to note that neither $u(t)$ nor $\dddot{G}(t)$ occurs in the "additional terms" $\left\{A d d_{1}, A d d_{2}, A d d_{3}, A d d_{4}\right\}$.

If the designer wishes to use a model-based controller he/she has to take the burden of the calculation of the additional terms for which precise knowledge of the model parameters and at least the estimated state variables are needed. Instead of that the FPI-based adaptive controller is satisfied with the rough and simple affine model in (2) as

$$
u(t) \approx-\frac{V_{I} t_{\max , I}^{2} \dddot{G}(t)}{K_{x g i} G_{0}}+\beta,
$$

in which instead of $G(t)$ a constant value $G_{0}=12 \mathrm{mM} \cdot \mathrm{L}^{-1}$ is written, and instead of the time- and delay-dependent additional term a constant value $\beta=10^{-3} \mathrm{pM} \cdot \mathrm{kg}^{-1} \cdot \mathrm{min}^{-1}$

\begin{tabular}{|c|c|}
\hline Physical Quantity & Value \\
\hline Glucose concentration in plasma $G\left[m M \cdot L^{-1}\right]$, & variable \\
\hline Insulin concentration in plasma $I\left[p M \cdot L^{-1}\right]$ & variable \\
\hline $\begin{array}{l}\text { Insulin mass in the accessible } \\
\text { subcutaneous depo } S_{1}\left[\mathrm{pM} \cdot \mathrm{kg}^{-1}\right]\end{array}$ & variable \\
\hline $\begin{array}{l}\text { Insulin mass in the not accessible } \\
\text { subcutaneous depo } S_{2}\left[\mathrm{pM} \cdot \mathrm{kg}^{-1}\right]\end{array}$ & variable \\
\hline $\begin{array}{l}\text { The subcutaneous insulin delivery } \\
\text { rate (control input) } u\left[\mathrm{pM} \cdot \mathrm{min}^{-1} \cdot \mathrm{kg}^{-1}\right]\end{array}$ & variable \\
\hline $\begin{array}{l}\text { The rate of glucose uptake } \\
\text { by tissues } K_{x g i}\left[L \cdot p M^{-1} \cdot \mathrm{min}^{-1}\right]\end{array}$ & $3.11 \times 10^{-5}$ \\
\hline $\begin{array}{l}\text { The net balance between hepatic glucose output } \\
\text { and insulin-independent zero-order glucose } \\
\text { tissue uptake } T_{g h}\left[\mathrm{mM} \cdot \mathrm{kg}^{-1} \cdot \mathrm{min}^{-1}\right]\end{array}$ & 0.003 \\
\hline $\begin{array}{l}\text { The apparent distribution volume } \\
\text { for glucose } V_{G}\left[L \cdot \mathrm{kg}^{-1}\right]\end{array}$ & 0.187 \\
\hline $\begin{array}{l}\text { The apparent first-order disappearance rate } \\
\text { constant for insulin } K_{x i}\left[\mathrm{~min}^{-1}\right]\end{array}$ & $1.211 \times 10^{-2}$ \\
\hline $\begin{array}{l}\text { The maximal rate of second-phase insulin } \\
\text { release } T_{i G \max }\left[\mathrm{pM} \cdot \mathrm{kg}^{-1} \cdot \mathrm{min}^{-1}\right]\end{array}$ & 1.573 \\
\hline $\begin{array}{l}\text { The apparent distribution volume } \\
\text { for insulin } V_{I}\left[L \cdot \mathrm{kg}^{-1}\right]\end{array}$ & 0.25 \\
\hline $\begin{array}{l}\text { The time-to-maximum } \\
\text { insulin absorption } t_{\max , I}[\mathrm{~min}]\end{array}$ & 55 \\
\hline $\begin{array}{l}\text { The apparent delay with which the pancreas } \\
\text { varies secondary insulin release in response to } \\
\text { varying plasma glucose concentrations } \tau_{g}[\mathrm{~min}]\end{array}$ & 24.0 \\
\hline $\begin{array}{l}\text { The glycemia at which the insulin release } \\
\text { is half of its maximal rate } G^{\star}\left[m M \cdot L^{-1}\right]\end{array}$ & 9.0 \\
\hline $\begin{array}{l}\text { The progressivity with which the pancreas } \\
\text { reacts to circulating glucose concentrations } \\
\gamma[\text { nondimensional }]\end{array}$ & 3.205 \\
\hline
\end{tabular}
was written. This model even does not require a precise estimation of the parameters $K_{x g i}, V_{I}$, and $t_{\max , I}$. However, it requires the real-time measurement of $G(t)$, and on this basis, the numerical estimation of $\dddot{G}(t)$.

TABLE I

THE VARIABLES AND PARAMETERS OF THE DDE MODEL

If we prescribe a kinematic tracking strategy that determines a "desired" $\dddot{G}^{\text {Des }}(t)$ value so that guarantees the $G(t) \rightarrow G_{\text {ref }}$ convergence as $t \rightarrow \infty$ if it is precisely realized, the control task was successfully solved. For this purpose we can introduce a nominal trajectory $G^{N}(t)$ as it was done in [21] as given in (3) with a constant parameter $\tau_{\text {track }}>0$

$$
G^{N}(t)=G_{r e f}+\left(G_{i n i}-G_{r e f}\right) \exp \left(\frac{-\left(t-t_{i n i}\right)}{\tau_{\text {track }}}\right)
$$


the various derivatives of $G^{N}(t)$ can be easily computed. By the use of a constant $\Lambda>0$ value for the tracking error we can prescribe that let be $e(t)=\left[G^{N}(t)-G(t)\right]$ the tracking error, and

$$
\left(\Lambda+\frac{\mathrm{d}}{\mathrm{d} t}\right)^{4} e(t) \equiv 0
$$

-here $G(t)$ is the realized glucose concentration-, that yields the kinematic tracking strategy

$$
\begin{aligned}
& \dddot{G}^{D e s}(t)= \\
& \dddot{G}^{N}(t)+\Lambda^{4} e(t)+4 \Lambda^{3} \dot{e}(t)+6 \Lambda^{2} \ddot{e}(t)+4 \Lambda \dddot{e}(t) .
\end{aligned}
$$

The solution of (5) is a polynomial function of $t$ multiplied with an exponentially damping term that guarantees that the tracking error will converge to 0 . The task of the adaptive controller is the realization of this strategy as precisely as possible. In the next section the adaptive controller's basic idea is outlined.

\section{The FPI-BASED AdAPTIVE CONTROLler}

The idea of this controller is the use of the approximate model (2) for the calculation of the necessary control signal $u(t)$ to realize (5). This corresponds to the use of an experimentally, real -time observable response function that yields the realized 4 th time-derivative as a function of the input of the approximate affine model. Since $\dddot{G}(t)$ can be instantaneously set by the controller, this function only slowly drifts since it depends on the actual state variables and their derivatives that lower order than $4: \dddot{G}(t)=\Phi\left(\dddot{G}_{i n p}(t)\right)$. Since our model was very imprecise $\dddot{G}(t) \neq \dddot{G}_{\text {inp }}(t)$, so in the place of $\dddot{G}^{D e f}(t)$ an iterative sequence is so written that in each digital control step only one step of this iteration can be realized (Fig. 1). If the iteration is well set $\dddot{G}^{D e f}(t) \rightarrow$ $\dddot{G}_{\star}^{\text {Def }}$ that yields $\dddot{G}^{\text {Des }}=\Phi\left(\dddot{G}_{\star}\right)$. (As the state variables drift, $\dddot{G}_{\star}$ also slowly drifts.) If the convergence of the sequence is fast enough, the single iterative step during one digital control cycle may provide good results.

For the creation of the necessary sequence Banach's Fixed Point Theorem [23] is used that states that in a linear, normed, complete metric space the contractive $\Psi$ maps in the form $\left\{\ldots x_{n+1}=\Psi\left(x_{n}\right), \ldots\right\}$ create Cauchy sequences that due to the completeness of the space converge to a limit point in the space $x_{\star}$ so that it is the uniquely determined fixed point of this function as $x_{\star}=\Psi\left(x_{\star}\right)$. That is in the first step the control task was transformed into a fixed point problem the appropriate map of which in the second step was made contractive, and finally the problem was solved via iteration. This method has very old antecedents (e.g. [24][27]), for instance the proof of the Picard-Lindelöf theorem (e.g. [28]) was based on the basis of similar considerations.

For instance, a real differentiable function $\Phi(x)$ is contractive, if $\exists 0 \leq K<1$ so that $\left|\Phi^{\prime}(x)\right| \leq K$. In [29] for the iteration described in Fig. 1 the function in (6) was used

$$
\begin{aligned}
& \dddot{q}_{n+1}^{D e f}=\left(K_{c}+\dddot{q}_{n}^{D e f}\right) . \\
& \cdot\left[1+B_{c} \tanh \left(A_{c}\left(\Psi\left(\dddot{q}_{n}^{D e f}\right)-\dddot{q}_{n+1}^{D e s}\right)\right)\right]-K_{c},
\end{aligned}
$$

having only three adaptive control parameters $K_{c}, A_{c}, B_{c}$. For making it convergent this mapping must be made contractive in the vicinity of the fixed point. The derivative of this function in $\dddot{G}_{\star}$ is

$$
\left.\frac{\mathrm{d} \dddot{G}_{n+1}^{D e f}}{\mathrm{~d} \dddot{G}_{n}^{D e f}}\right|_{\dddot{G}_{\star}}=1+\left.\left.B_{c} \frac{\mathrm{d} \tanh (x)}{\mathrm{d} x}\right|_{0} A_{c} \frac{\mathrm{d} \Psi(r)}{\mathrm{d} r}\right|_{\dddot{G}_{\star}}
$$

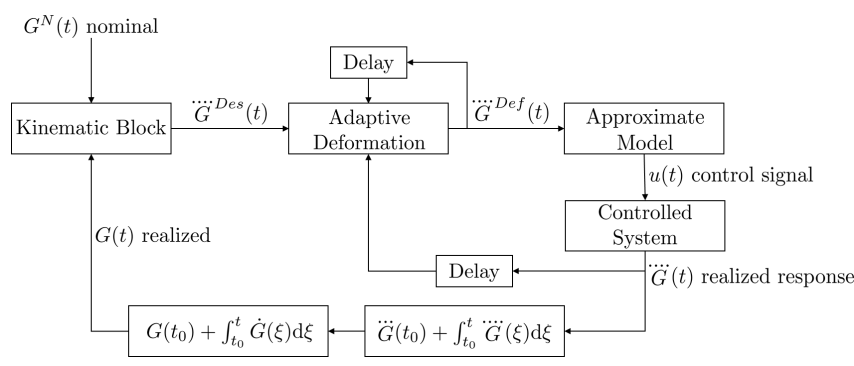

Fig. 1. The structure of the "Fixed Point Transformation-based Adaptive Controller" - modification of the figure in [22]

To achieve contractivity it is expedient to use a big $K_{c}>>$ $\left|\dddot{G}^{D e f}\right|$ control parameter, $B_{c}= \pm 1$, and a small positive parameter $A_{c}$ to achieve that $-1<\left.\frac{\mathrm{d} \dddot{G}_{n+1}^{D e f}}{\mathrm{~d} \dddot{G}_{n}^{\text {Def }}}\right|_{\dddot{G}_{\star}}=1-$ $\varepsilon<1$ in which $\varepsilon$ is a small positive number. The method has the practical value that for achieving convergence not very precise setting of these parameters is needed. The speed of convergence, consequently the tracking precision of the controller slightly depends on these parameters, too. It worths noting that this controller also feeds back the observed fourth time-derivative as e.g. in the control of Classical Mechanical systems the Acceleration Feedback Controllers (e.g. [31][33]) feeds back the 2nd derivaives, but in a different manner. Though this signal may be very noisy, the success of these controllers anticipates that the noise problems can be tackled in the case of the fixed point iteration-based controllers.

For the observation of $\dddot{G}$ the following "heuristic polynomial differentiator" was applied: in each control cycle the last $2 N+1$ measured values of $\hat{G}$ observed in the discrete time grid was mapped to the integer grid-points $\{-N,-N+$ $1, \ldots, 0, \ldots, N\}$ as $\{\tilde{G}(-N), \ldots, \tilde{G}(N)\}$, then an order 4 polynomial was fitted to these values by minimizing the approximation error $\sum_{k=-N}^{N}\left(\sum_{\ell=0}^{4} c_{\ell} k^{\ell}-\tilde{G}(k)\right)^{2}$. This method heuristically considers the variation of the signal that is "more hectic" than that of the order 4 polynomial as "noise" and filters it out. Then the derivatives of the polynomial were analytically calculated by the use of the rule $\left(k^{\ell}\right)^{\prime}=\ell k^{(\ell-1)}$. Finally, by the use of the chain rule of differentiation these derivatives were mapped back to the actual time-grid. Though this method realizes some noise 
filtering, too, we do not state that its filtering effect is "strong enough" for compensating the effects of the measurement noises in our particular case. However, this method simply can be combined with other, traditional and well applicable noise filtering methods. As in [21], the noise filtering remained an open question that needs further investigations.

\section{Simulation Results}

The simulations have carried out by using Julia ${ }^{\mathrm{TM}}$ v1.1.0. and PyPlot package was applied to generate the diagrams.

In the simulations the following fine details were applied. For considering the range $G_{i n i} \in[2,13] m M \cdot L^{-1}$ various $\Lambda_{0}$ parameters were used in (5) according to the rule as follows (in Julia code):

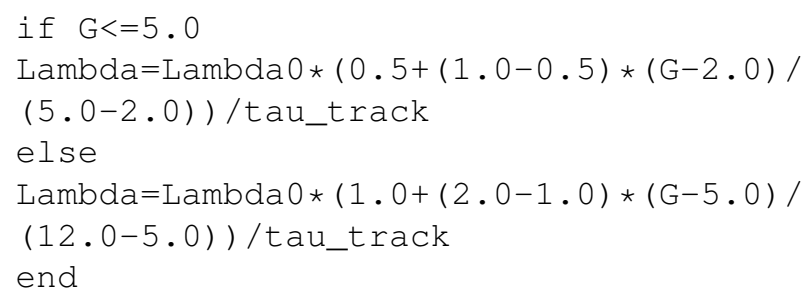

The time resolution of the digital controller was $\delta t=5 \mathrm{~min}$ (it means the duration of the digital control cycle during which the control signal $u$ is constant); the time-resolution of the Euler integration applied during each control cycle was $\delta \delta t=\delta t / 50 ;$ as in [21], for the construction the nominal trajectory $\tau_{\text {track }}=100 \mathrm{~min}$ was used in (3). The adaptive control parameters were set as $A_{c}=255 \mathrm{~L} \cdot \mathrm{min}^{4} \cdot \mathrm{mM}^{-1}$, $K_{c}=10^{-4} \mathrm{mM} \cdot L^{-1} \cdot \mathrm{min}^{-4}, B_{c}=-1$. The control signal $u_{1}$ obtained from the affine model (2) was maximized according to the rule $u=u_{\max } \tanh \left(u_{1} / u_{\max }\right)$ with $u_{\max }=$ $0.18 \mathrm{pM} \cdot \mathrm{min}^{-1} \cdot \mathrm{kg}^{-1}$. When negative control signal was calculated, it was truncated at 0 due to phenomenological considerations, and the adaptivity of the control was switched off in the lack of available signal variation. (It is switched on again, as the truncation is over.) The initial state variables were $I_{i n i}=T_{g h} /\left(V_{G} K_{x g i} G_{i n i}\right), S_{1 i n i}=S_{2 i n i}=0$.

Figures 2, 3, and 4 reveal the state varaibles, the 4 th derivatives of $G(t)$ and the control signal $u(t)$ for a very high initial glucose concentration $G_{i n i}=16.0 \mathrm{mM} \cdot L^{-1}$ and $\Lambda_{0}=2.5 \mathrm{~min}^{-1}$ that was found "ideal" in the sense that it did not cause too much truncation at 0 and saturation at $u_{\max }$ in $u(t)$. Approximately during 2 hours the gulcose concentration was lessened under the critical level of $11.0 \mathrm{mM} \cdot \mathrm{L}^{-1}$, and after about 8 hours it was stabilized around $G_{r e f}$. The adaptivity worked well, the "desired" and the "realized" 4th derivatives slowly converged to each other and considerably differred from the "deformed" ones.

Figures 5, 6, and 7 testify that even a considerable increase in $\Lambda_{0}$ from 2.5 to $4.0 \mathrm{~min}^{-1}$ does not have too significant effects: truncations appear at $u=0$, and the built-in saturation at $u_{\max }$ is approached several times. Consequently in certain intervals the adaptive deformation was switched off. Figures 8 and 9 belong to the "ideal" $\Lambda_{0}=2.5 \mathrm{~min}^{-1}$ and a very low initial glucose concentration level $G_{i n i}=$ $2.0 \mathrm{mM} \cdot L^{-1}$. The "reference value" was stabilized about

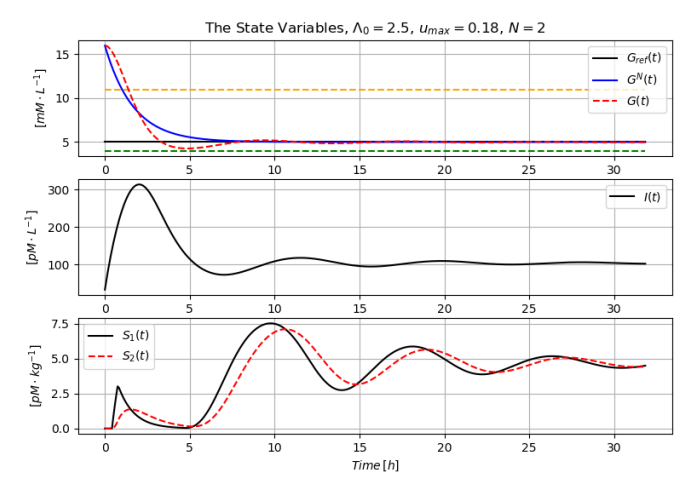
Fig. 2. The state variables for $G_{i n i}=16.0 \mathrm{mM} \cdot L^{-1}$ and $\Lambda_{0}=$
$2.5 \mathrm{~min}^{-1}$

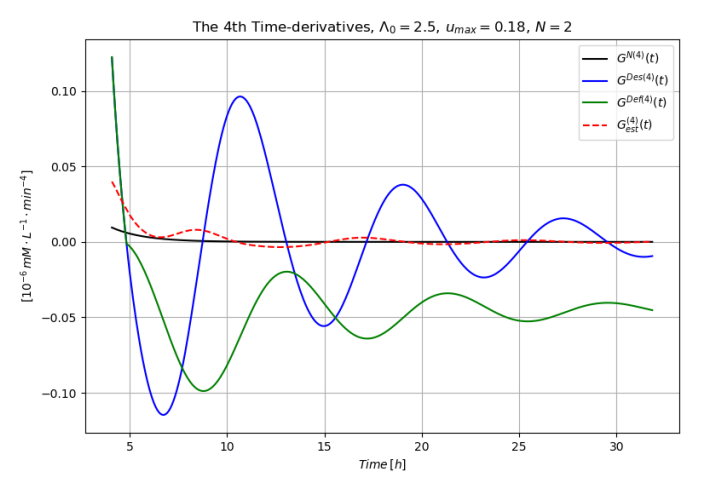

Fig. 3. The $\dddot{G}$ derivatives for $G_{i n i}=16.0 \mathrm{mM} \cdot L^{-1}$ and $\Lambda_{0}=$ 2.5 min $^{-1}$

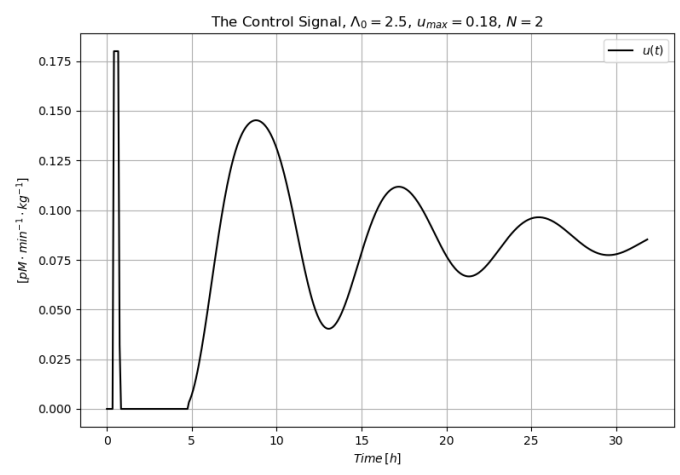

Fig. 4. The control signal $u(t)$ for $G_{i n i}=16.0 \mathrm{mM} \cdot L^{-1}$ and $\Lambda_{0}=$ 4.0 min $^{-1}$

after 6 hours. Figure 10 shows that the drastic increase in $\Lambda_{0}$ again causes truncations and saturations in $u(t)$, limits the length of the adaptive sessions, however, its effect is not critical.

\section{CONCLUSIONS}

In this paper a Fixed Point Iteration-based adaptive solution was suggested to adaptively control the insulin ingress rate in treating the illness Diabetes Mellitus. In this study, we did not consider the usage of continuous glycemic 


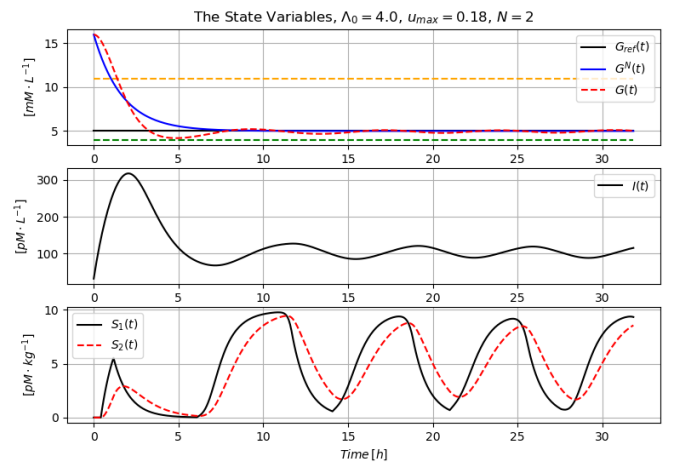

Fig. 5. The state variables for $G_{i n i}=16.0 \mathrm{mM} \cdot L^{-1}$ and $\Lambda_{0}=$ 4.0 min $^{-1}$

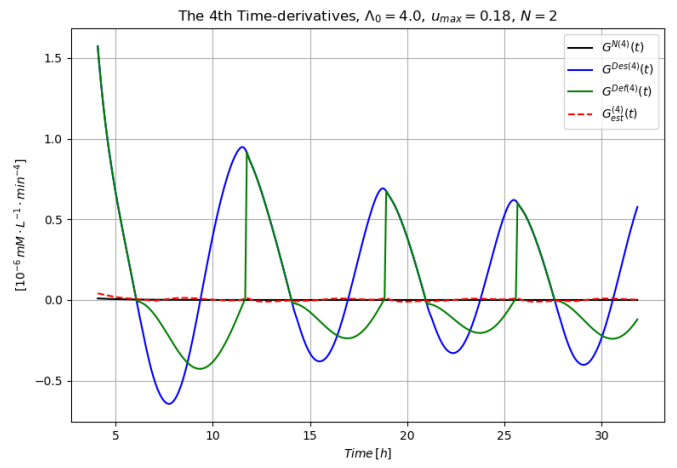

Fig. 6. The $\dddot{G}$ derivatives for $G_{i n i}=16.0 \mathrm{mM} \cdot \mathrm{L}^{-1}$ and $\Lambda_{0}=$ $4.0 \mathrm{~min}^{-1}$

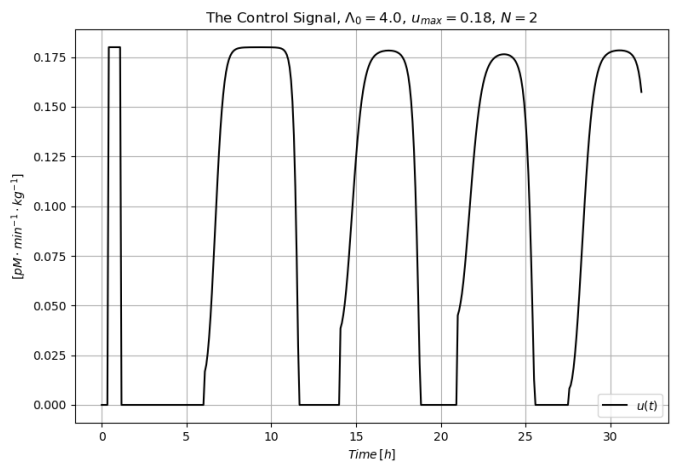

Fig. 7. The control signal $u(t)$ for $G_{i n i}=16.0 \mathrm{mM} \cdot \mathrm{L}^{-1}$ and $\Lambda_{0}=$ $4.0 \mathrm{~min}^{-1}$

measurement system. It was only assumed that the blood glucose level is directly accessible for the sake of simplicity, since our goal was to prove the controller device concept. In contrast to the precise model-based control that uses state observation technique this simple approach utilizes only a very simple affine model of the controlled phenomenon. Instead calculating the necessary information from the model a simple order 4 polynomial differentiator is applied to solve the control task which has 4 relative order. The operation of

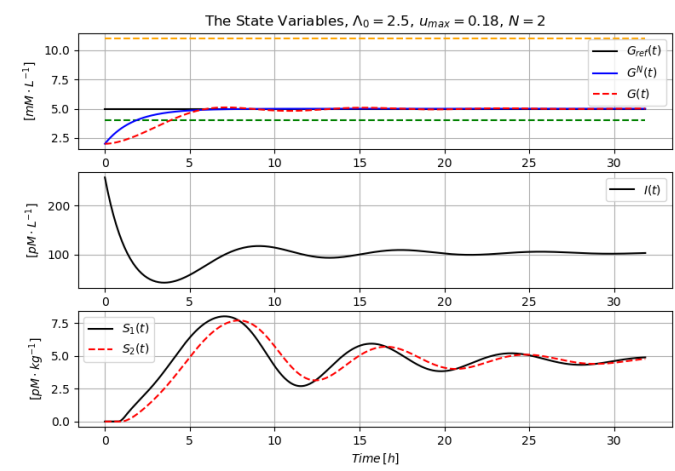
Fig. 8. The state variables for $G_{i n i}=2.0 \mathrm{mM} \cdot L^{-1}$ and $\Lambda_{0}=$
$2.5 \mathrm{~min}^{-1}$

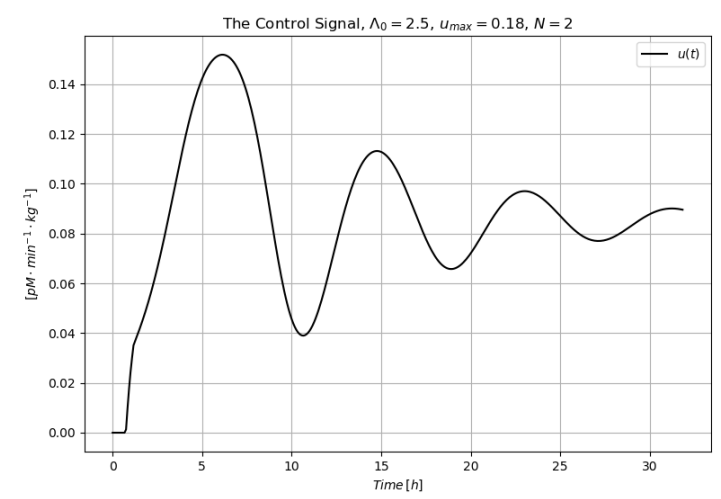

Fig. 9. The control signal $u(t)$ for $G_{i n i}=2.0 \mathrm{mM} \cdot L^{-1}$ and $\Lambda_{0}=$ $2.5 \mathrm{~min}^{-1}$

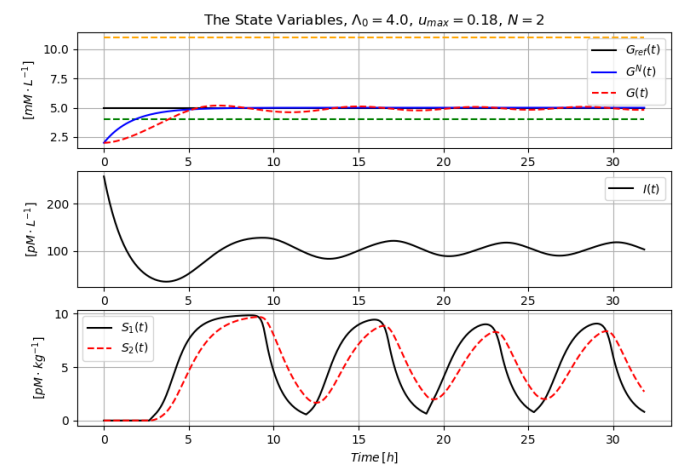
Fig. 10. The state variables for $G_{i n i}=2.0 \mathrm{mM} \cdot L^{-1}$ and $\Lambda_{0}=$
$4.0 \mathrm{~min}^{-1}$

this controller was numerically investigated in integer steps in the range of the initial glucose concentration $G_{i n i} \in$ $[2,16] m M \cdot L^{-} 1$. The simulation results are comparable with that published in [21]. In the further work we wish to investigated and tackle the problem of the observation noises.

\section{REFERENCES}

[1] M.M.R.F. Struys, H. Vereecke, A. Moerman, E.W. Jensen, D. Ver- 
haegen, N. De Neve, F.J.E. Dumortier, and E.P. Mortier, "Ability of the Bispectral Index, autoregressive modelling with exogenous input-derived auditory evoked potentials, and predicted Propofol concentrations to measure patient responsiveness during anesthesia with Propofol and Remifentanil," Anesthesiology, vol. 99, no. 4, pp. 802812, 2003.

[2] T. Zikov, S. Bibian, G.A. Dumont, M. Huzmezan, and C.R. Ries, "Quantifying Cortical Activity During General Anesthesia Using Wavelet Analysis," IEEE Transactions on Biomedical Engineering, vol. 53, no. 4, pp. 617-632, 2006.

[3] R.N. Bergman, Y.Z. Ider, C.R. Bowden, and C. Cobelli, "Quantitative estimation of insulin sensitivity," Am. J. Physiol. Endocrinol. Metab., vol. 236, 199, pp. E667-E677.

[4] C. Dalla Man, R.A. Rizza, and C. Cobelli, "Meal simulation model of glucose-insulin system," IEEE Transactions on Biomedical Engineering, vol. 54, no. 10, pp. 1740-1749, 2007.

[5] J. Bondia, S. Romero-Vivo, B. Ricarte, and J.L. Diez, "Insulin Estimation and Prediction: A Review of the Estimation and Prediction of Subcutaneous Insulin Pharmacokinetics in Closed-Loop Glucose Control,” IEEE Contr Syst, vol. 38, no. 1, pp. 47-66, 2018.

[6] P. Palumbo, S. Panunzi, and A. De Gaetano, "Qualitative behavior of a family of delay differential models of the glucose insulin system," Discrete Contin. Dyn. Syst.-Ser. B, vol. 151, pp. 399-424, 2007.

[7] S. Panunzi, P. Palumbo, and A. De Gaetano, "A discrete single delay model for the Intra-Venous Glucose Tolerance Test," Theor. Biol. Med. Model., vol. 4, pp. 1-16, 2007.

[8] J. Artigas and G. Hirzinger, "A Brief History of DLR's Space Telerobotics and Force-Feedback Teleoperation," Acta Polytechnica Hungarica, vol. 13, no. 1, pp. 239-249, 2016.

[9] Y. Zhang and C. Yi, Zhang Neural Networks and Neural-Dynamic Method, Nova, New York, 2011.

[10] Y. Zhang, Y. Chou, J. Chen, Z. Zhang, and L. Xiao, "Presentation, Error Analysis and Numerical Experiments on a Group of 1-stepahead Numerical Differentiation Formulas," J. Comput. Appl. Math., vol. 239, pp. 406-414, 2013.

[11] L. Jin and Y. Zhang, "Continuous and Discrete Zhang Dynamics for Real-time Varying Nonlinear Optimization," Numer. Algorithms, vol. 73, no. 1, pp. 115-140, 2015

[12] Qing-Kui Li, Jun Zhao, and Georgi M. Dimirovski, "Robust tracking control for switched linear systems with time-varying delays," IET Control Theory and Applications, vol. 2, no. 6, pp. 449-457, 2008.

[13] Jie Lian, Jun Zhao, and Georgi M. Dimirovski, "Robust Hinf sliding mode control for a class of switched delay systems," International Journal of Systems Science, vol. 40, no. 8, pp. 855-866, 2009.

[14] Qing-Kui Li, Jun Zhao, and Georgi M. Dimirovski, "Tracking control for switched time-varying delay systems with stabilizable and unstabilizable subsystems," Nonlinear Analysis: Hybrid Systems, vol. 3, no. 2, pp. 133-142, 2009.

[15] Bin Yang, Rui Wang, Peng Shi, and Georgi M. Dimirovski, "New delay dependent stability criteria for recurrent neural network with time-varying delays," Neurocomputing, vol. 151, no. P3, pp. 1414$1422,2015$.

[16] R.E. Kalman, "A New Approach to Linear Filtering and Prediction Problems," Transaction of the ASME - Journal of Basic Engineering, vol. 82(Series D), pp. 35-45, 1960.

[17] D.G. Luenberger, Introduction to Dynamic Systems: Theory, Models, and Applications, John Wiley \& Sons, New York, 1979.

[18] J. Zhang and Z. Zhang, Application of a Strong Tracking FiniteDifference Extended Kalman Filter to Eye Tracking - Lecture Notes in Computer Science Vol. 4113/2006 - Intelligent Computing,' SpringerVerlag Berlin, Heidelberg, Germany, 2006.

[19] A. Germani, C. Manes, and P. Pepe, "An asymptotic state observer for a class of nonlinear delay systems," Kybernetika, vol. 36, pp. 459-478, 2001.

[20] A. Germani, C. Manes, and P. Pepe, "A state observer for a class of nonlinear systems with multiple discrete and distributed time delays," Eur. J. Control, vol. 11, pp. 196-205, 2005.

[21] P. Palumbo, P. Pepe, S. Panunzi, and A. De Gaetano, "Observer-based glucose control via subcutaneous insulin administration," In Proc. of the 8th IFAC Symposium on Biological and Medical Systems, August 29-31, 2012, Budapest, Hungary, pp. 107-112, 2012.

[22] H. Redjimi and J.K. Tar, "On the effects of time-delay on precision degradation in Fixed Point Transformation-based adaptive control," In the Proc. of the 2017 IEEE 30th Jubilee Neumann Colloquium, November 24-25, 2017, Budapest, Hungary, 2017, pp. 125-130.
[23] S. Banach, "Sur les opérations dans les ensembles abstraits et leur application aux équations intégrales (About the Operations in the Abstract Sets and Their Application to Integral Equations)," Fund. Math., vol. 22, pp. 133-181, 1922.

[24] Tjalling J. Ypma, "Historical development of the Newton-Raphson method," SIAM Review, vol. 37, no. 4, 1995, pp. 531-551.

[25] J.M. Ortega and W.C. Rheinboldt, Iterative Solution of Nonlinear Equations in Several Variables, SIAM, 2000.

[26] C.T. Kelley, Solving Nonlinear Equations with Newton's Method, no 1 in Fundamentals of Algorithms, SIAM, 2003.

[27] P. Deuflhard, Newton Methods for Nonlinear Problems. Affine Invariance and Adaptive Algorithms, Springer Series in Computational Mathematics, Vol. 35, Springer, Berlin, 2004

[28] V.I. Arnold, Ordinary Differential Equations, The MIT Press, 1978.

[29] J.K. Tar, J.F. Bitó, L. Nádai, and J.A. Tenreiro Machado, "Robust Fixed Point Transformations in adaptive control using local basin of attraction," Acta Polytechnica Hungarica, vol. 6, no. 1, 2009, pp. 2137.

[30] J.K. Tar, "Towards replacing Lyapunov's "Direct" Method in adaptive control of nonlinear systems" (invited plenary lecture at the Mathematical Methods in Engineering Intl. Symp. MME, 21-24 October 2010, Coimbra, Portugal), chapter in Mathematical Methods in Engineering (Eds.: N.M. Fonseca Ferreira \& J.A. Tenreiro Machado), Springer Dordrecht, Heidelberg, New York, London, 2014, pp. 35-45.

[31] Karl-Petter W. Lindegaard, Acceleration Feedback in Dynamic Positioning (PhD Dissertation), Department of Engineering Cybernetics, Norwegian University of Science and Technology, NO-7491 Trondheim, Norway, 2003.

[32] E. Dumetz, Jean-Yves Dieulot, Pierre-Jean Barre, F. Colas. and T. Delplace, "Control of an Industrial Robot using Acceleration Feedback," Journal of Intelligent and Robotic Systems, vol. 46, no. 2, pp. $111-128,2006$.

[33] Qiang Wang, Hua-Xiang Cai, Yong-Mei Huang, Liang Ge, Tao Tang, Yan-Rui Su, Xiang Liu, Jin-Ying Li, Dong He, Sheng-Ping Du, and Yu Ling, "Acceleration feedback control (AFC) enhanced by disturbance observation and compensation (DOC) for high precision tracking in telescope systems," Research in Astronomy and Astrophysics, vol. 16, no. 8, p. 124, 2016.

[34] Gy. Eigner and P. Horváth and J.K. Tar, I.J. Rudas and L. Kovács "Application of Robust Fixed Point control in case of T1DM" 2015 IEEE International Conference on Systems, Man, and Cybernetics, p. 2459-2464, 2015. 